\title{
Thinking Ecology for Architecture: Exploration of Cool Pocket
}

\author{
Rini Suryantini ${ }^{1, *}$, M. M. Y. Harahap ${ }^{1}$, Yandi Andri Yatmo $^{1}$, and Nandy Putra $^{2}$ \\ ${ }^{1}$ Department of Architecture, Faculty of Engineering, Universitas Indonesia, Kampus Universitas Indonesia Depok 16424, \\ Indonesia \\ 2 Department of Mechanical Engineering, Faculty of Engineering, Universitas Indonesia, Kampus Universitas Indonesia \\ Depok 16424, Indonesia
}

\begin{abstract}
This paper discusses the importance of understanding the ecology as part of architectural education. Rather than focusing on the heat phenomenon as frequently researched, this paper explores the opposite ideas by identifying the concept of cool pocket and designing it as a mean for heat mitigation in a tropical built environment. Recent researches show that providing a microclimate that responds human thermal comfort can contribute to the building energy performance and at the same time promote activities in outdoor space. The relation and interaction among various microclimate factors with the land, water body, arrangement of vegetations, building's geometry and configurations, create different scenarios of the thermal condition, including possible cool pocket. Using air temperature and wind as indicators, possibilities of cool pocket will be explored and investigated through CFD Simulation. The result shows the importance of ecological understanding as part of designing cool pocket in a tropical context in a learning process and scenario-based simulation in developing design strategies.
\end{abstract}

\section{Learning Ecology: Heat Island, Microclimate, and Cool Pocket}

Ecology is an inseparable part of architecture and understanding ecology is a necessity whether or not architectural sustainability is at the core of our concern. In the last decades, there has been a significant shift towards the development of integration among ecology, built environment and energy into architectural design [1]. This integration includes a discussion towards thermal comfort which affects human lives.

"...the task of architecture is not merely to abolish gross thermal extremes but to provide the optimal thermal environments for the whole spectrum of modern life" [2]. This, in other words, indicates that thermal condition is one of the main concerns of architecture. Thermal condition of the particular environment should be embedded in the architecture itself. It is not an object resulted in the architecture, but rather a dynamic engagement of elements of the ecological system.

Learning ecology particularly gives us a better perspective towards the built environment as it "...has potential to be an analytical tool, as ecology comprises relation and interaction in the complexity of systemic nature..." [3]. However, there is a question of how and in what way we can use ecology as an analytical tool since the system involves various aspects to it. Moreover, this question leads to another critical issue of

\footnotetext{
* Corresponding author: rinisuryantini@gmail.com
}

how do we incorporate ecological thinking into architectural design education. This paper will try to answer these questions through a scenario-based study using simulation which shows the relation and interaction in the ecological system. In particular, this paper imposes the local heat island effect phenomenon. The presence of heat within the built environment, its relation to microclimate and the mitigation are mainly the focus in the research of heat island phenomenon [46]. This paper offers the opposite ideas and explores the presence of cool area or pocket within a tropical environment as heat mitigation design. As part of an ecological system, the presence of warmth or cool will be simulated by reconfiguring the built environment with a specific setting and climatic attributes. The result of the study will eventually demonstrate architecture as the cool pocket. This strongly suggests that cool pocket is a simple yet effective concept to introduce and explain the ecological understanding in architectural design education.

\section{Designing Cool Pocket to Mitigate Heat Island Effect}

To mitigate the heat island effect, the relation and interaction among aspects related to human thermal comfort and microclimate within a context need to be recognised. The microclimate itself is responsible for the 
thermal condition within a context and consequently to the human comfort [7]. If thermal comfort is a condition defined by air temperature, air flow, relative humidity, mean radiant temperature, aside the human factor (metabolism rate and clothing), the microclimate is composed by climatic attributes - air temperature, air flow, humidity, solar radiation, heat release, etc - and their interaction with building arrangements, vegetation, and water body. These elements are interrelated and create a specific thermal condition. As an environmental phenomenon, thermal (including humidity and air movement) is one of the materials that is reckoned in spatial design that can create a certain atmosphere and spatial experiences. Mapping environment is one of the methods that can be developed to capture thermal as a way of knowing the environment [8]. By identifying the desired thermal condition, mitigation acts can be designed and thus the effect of the heat island effect can be reduced.

There are researches with various focuses that explore the heat island phenomenon, its effect and how to mitigate the impact. Among those researches are ones which focus is conducting calculation and simulation study on building envelope and its topological and geometrical properties [9-11], water body [12], and vegetation [13]. Each of these studies was conducted especially to see whether the heat island effect could be reduced through various level of intervention. The investigations resulted in possible mitigation, such as modifying building envelope and creating an oasis-like condition, which consequently changes the thermal condition of the studied environment. These modifications mainly manipulate the condition of the environment from heat island to a desired thermal condition, which further in this paper is going to be referred to as, cool pocket.

Building envelope can affect the thermal condition not only within the building but also outside the building itself. It implies that building envelope acts as the barrier which separates the natural (outside) and artificial (inside) thermal condition [11]. The envelope basically could be a tool to selectively create the desired thermal condition. By controlling the building envelope performance, an architect is as well benefited with a chance to mediate and manipulate the outside thermal condition. Through its topological formation, for example, building envelopes that are arranged close to one another could reduce the risk of local heat island effect [10]. Another aspect of the building envelope, such as height configuration, could affect the wind potential which consequently reduces local heat island effect.

Besides building envelope, other features that are present within a context contribute to the local climate as well. The features refer to vegetation and water body. Their configuration is affecting the thermal condition of the particular environment. Through certain arrangements, vegetation and water body could improve the mitigation of heat island effect, creating an oasis-like condition - a cooling effect. Arrangement and plantation of different types of vegetation could create certain wind turbulence and consequently enhance the quality of the space [13]. Meanwhile, a study also shows that water body which occurs either centralized or scattered around in a particular environment could either significantly create a cooling effect or distribute respectively temperature and humidity more even [12].

Overall, building envelope, vegetation and water body, through specific configuration acts could be considered as tools to manipulate or moreover design the desired thermal condition in particular environment, changing status from heat island to cool pocket. Retrospectively, the three aspects are believed as a proper representation of the environment to introduce ecological thinking in architectural design education.

\section{Method: Simulation to Learn the Cool Pocket Creation}

Approaching the Education 4.0, where experiential learning is aimed, the dynamic technology layer will play an important role and provide the students with more options and flexibility [14]. Computational Fluid Dynamics (CFD) provides those options and flexibility in the field of design and research, introducing environments that can be designed and observed [15]. By incorporating CFD simulation to the early stages of architectural design, the students can learn to decide responsibly and proposed design that based on evidence [15-17].

Through the development of computational tools, various techniques of observation and simulation have been developed to study the heat island phenomenon [16]. By providing solutions through the field distribution of pressure, air velocity, temperature, the concentration of water vapour (relative humidity) and contaminants, and turbulence [1], CFD can generate models simultaneously and accurately that describes the heat phenomenon. Particularly in this study, climatic attributes such as temperature and air flow, as driving factors of heat formation in outdoor space [18], will be explored through a scenario-based CFD simulation.

The proposed scenario-based simulation is then again argued to promote the flexibility in architectural learning. It offers various possible scenarios that show dynamic relation and interaction of elements within a context and thus develop the ecological awareness in architectural education. The SketchUp 3D Modeling software is used for architectural modelling, while CFD software from the Autodesk for the simulation and analysis.

In this paper, the study simulates an existing building located in a tropical campus environment in Depok, Indonesia. This building is characterised by its " $\mathrm{A}$ "-like shape with a courtyard within. With the tropical climate as its context, this courtyard is often indicated especially for its uncomfortable thermal condition. In order to show the indication, a simulation is run. Since humidity plays a role in tropical context, it is necessary not only to incorporate air temperature but also air flow in the simulation to overcome the humidity and create the desired thermal condition. The cool air temperature and rather high air velocity will be used as indicators of cool 
pocket. After the existing condition is acquired, it is then followed by two consecutive simulation stages : 1) A simulation study which parallelly investigates how the configuration of each element: vegetation and water body could mitigate the heat island condition in the courtyard, and 2) An advance simulation which responds the result of the first category. In this category, scenarios are developed by incorporating the building geometry configuration.

\section{In Search of Cool Pocket}

The study is started by simulating the existing building courtyard to have a preliminary understanding of the current thermal condition. This near the ground condition resulted from the simulation shows that the existing geometrical configuration of the building causes limited air movement within the courtyard and creates several heat pockets in the space (see Figure $1 \mathrm{~b}$ and 1c). Since the airflow from the space cannot remove the heat (and humidity), the uncomfortable condition is justified. Reflecting upon the initial simulation's results, it is learned that to mitigate the heat island; the airflow needs to be manipulated so that the heat is no longer trapped in the courtyard space and the overall the thermal condition can be improved as well.

\section{EXISITING CONDITION}

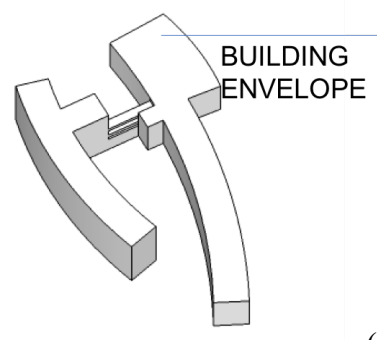

(a)

TEMPERATURE (CELCIUS)

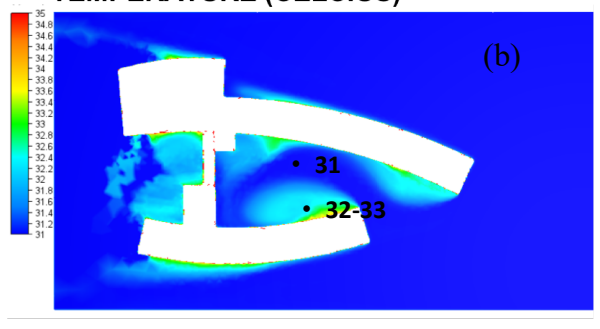

VELOCITY MAGNITUDE (M/S)

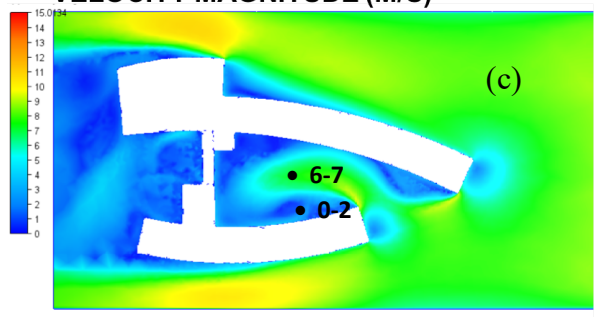

Fig. 1. Initial simulation of the existing building configuration (a) that shows the condition of temperature (b) and air flow (c)

\subsection{Vegetation and Water Body}

As suggested [12,13], the presence and arrangement of vegetation and water body can be used to affect the wind and temperature condition. In order to understand how vegetation and water body configuration affect the wind and temperature condition of the courtyard space separate simulations are performed. Simple configuration for each environment attributes is proposed to identify the thermal effect of each configuration.

The vegetation scenario is intended to have an insight of thermal condition from different arrangements of the vegetation. Originally, the idea is to position trees and learn how it affects the local climatic condition of the building's courtyard space. The Vegetation Scenario 1 places and arranges the vegetation confronting the building (see Figure 2a), meanwhile Vegetation Scenario 2 inside the courtyard (see Figure 2b). But, the trees with the configuration as seen as Vegetation Scenario 1 in Figure 2a, barricade the wind coming towards the building (from the right side) and do not directly affect thermal condition the courtyard space. The situation remains similar to the existing condition. Accordingly, another simulation - Scenario Vegetation 2 is performed. This simulation adds several trees within the courtyard space (see Figure 2b). This configuration results to be less windy in the courtyard space, although it lessens the heat pocket, distribute the air flow to a different area in the courtyard and consequently improves the temperature of the courtyard space (see Figure $2 b$ ).
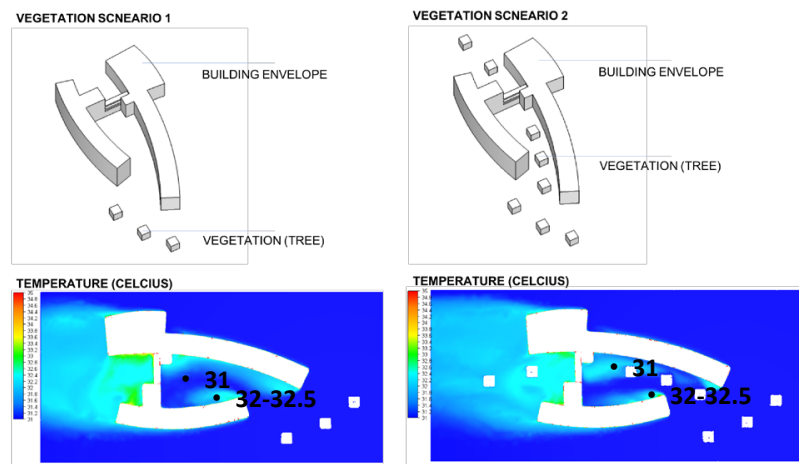

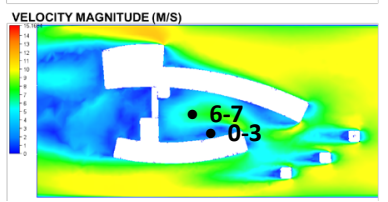

(a)

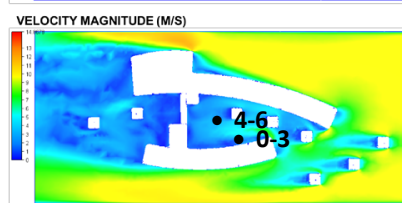

(b)
Fig. 2. Simulation's results that show temperature (above) and air flow (below) condition based on different tree configuration

Meanwhile, other simulations are performed to see how much the presence of water body would affect the thermal condition of the building's courtyard space. The simulations explore the effect of scattered and centralised configurations of the water body. Three scenarios on water body are prepared: Water Scenario 1 - placement of scattered small water bodies in the courtyard (see Figure 3a), Water Scenario 2 - placement 
combination of small water bodies and a larger water body in the courtyard (see Figure 3b), and Water Scenario 3 - placement of large water body toward the upcoming wind area (see Figure 3c). The results of the three scenarios show similar results [13] that the presence of centralised water body indeed significantly improves the temperature of the space.

Learned from Water Body Scenario 1 and 2 is that the larger the water body placed in the courtyard, the temperature is cooler above and surround the water body (see Figure $3 \mathrm{a}$ and $3 \mathrm{~b}$ ). The placement of the water body itself is then meaningful if it is evenly distributed in the courtyard. In addition, the scattered configuration of the water body is more effective on decreasing the temperature when they are placed as close as possible to the corner areas in the courtyard instead at the middle of it, although it can also reduce the air flow velocity due to evaporation resulted by large water body.
WATER BODY SCNEARIO 1
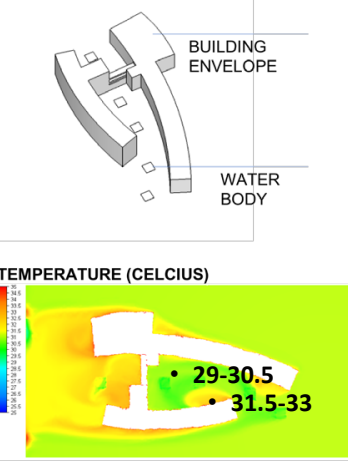

VELOCITY MAGNITUDE (M/S)

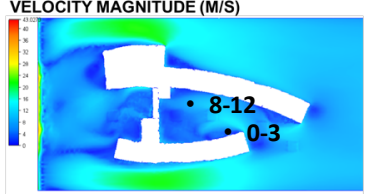

(a)
WATER BODY SCNEARIO 2
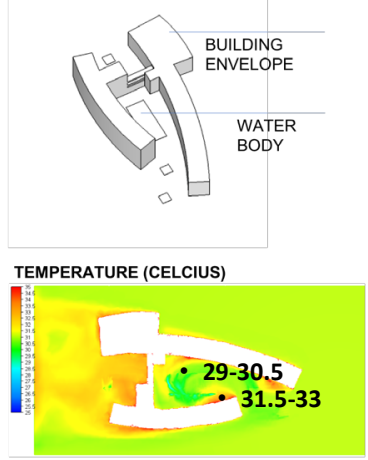

VELOCITY MAGNITUDE (M/S)

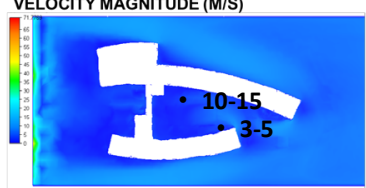

(b)
WATER BODY SCNEARIO 3
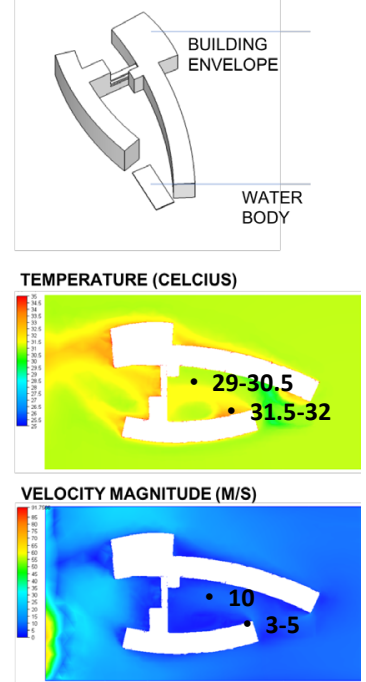

(c)

Fig. 3. Simulation's results that show temperature and air flow condition based on different water body configuration
From two scenarios, it is learned that the entering temperature is still high and therefore the Water Scenario 3 is run. As shown in Figure 3c, placing water body facing directly towards the upcoming wind decreases the temperature of the courtyard space. This can benefit the thermal condition as the air enters the courtyard. The air is cooled before it enters the courtyard and this shows that the large water body confronting the building can function reasonably as a buffer for hot air, although it still has its risk of water vapour or high humidity as well.

Overall, both vegetation and water body affect the thermal condition of the building's courtyard space, respectively towards its wind flow and temperature. This consequently could help us further on manipulating the heat island effect on the building's courtyard space into a cool pocket effect.

\subsection{Towards Cool Pocket}

Reflecting on the simulation results from the previous stage, advancing simulations are performed. This time, the aim is to explore the possibility of manipulating the building's geometry with the addition of vegetation and water body configuration. Particularly the vegetation, the trees are now positioned within the courtyard space as a windbreaker so that the breeze could reach the corner area of the courtyard space. Meanwhile, the water bodies are placed in the corner area and the frontline facing the upcoming wind. This aims respectively to spread the decrease in temperature and reduce the temperature of the coming air.

Those configurations are applied to three different geometry of the building's envelope scenarios. Figure 4 shows three scenarios, which are : Envelope Scenario 1 using the current building geometry (see Figure 4a), Envelope Scenario - 2 applying building geometry with subtracted volume in the middle of the building (see Figure 4b), and Envelope Scenario 3 - applying subtracted volume both in the middle and at the two edges of the building (see Figure 4c). Three building geometries are simulated to see how the design and manipulation of the existing building geometry could enhance the desired temperature in the courtyard space. In this case, the building mediates the thermal condition in the courtyard by providing a passage for the air flow.

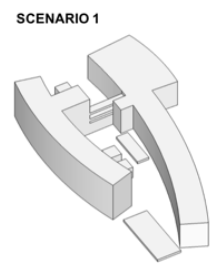

(a)

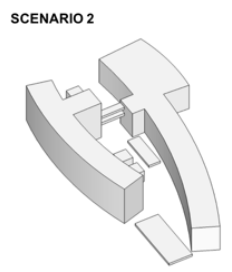

(b)

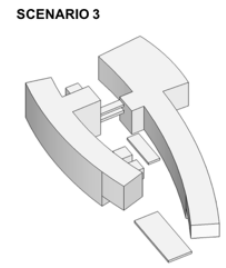

(c)
Fig. 4. Three envelope scenarios of building envelope

The simulation results (see Figure 5) of each of the above three configurations overall indicate that the subtracted volume improve the thermal condition of the building's courtyard space. In particular, the effect of the 
second building's envelope geometry configuration is that the wind is no longer trapped in the courtyard but flowing through from the courtyard to the back of the building. The observation is conducted in two level of views - near ground observation (ça $1.5 \mathrm{~m}$ above the ground level).

\section{ENVELOPE SCENARIO 1}
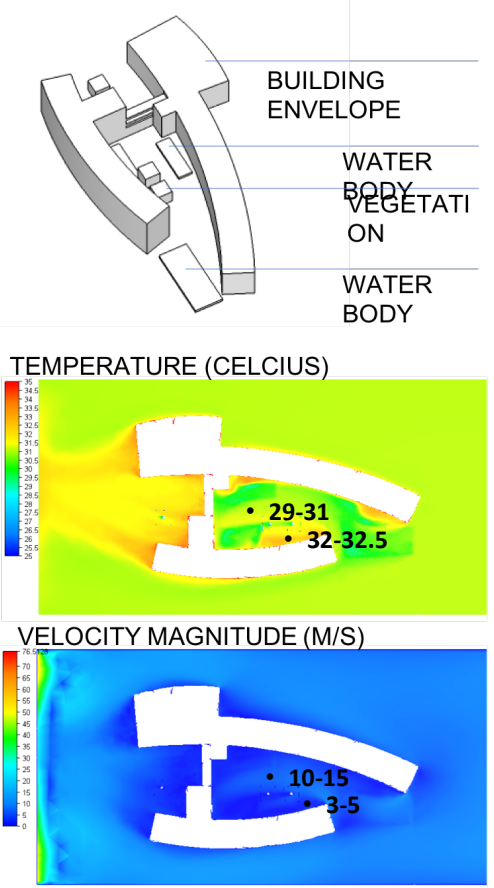

Fig. 5. Scenario 1 simulation based on first building envelope

Envelope Scenario 1 resulted in an improved thermal condition, which shows less area with higher temperature, although airflow remains similar. This happens due to the stable condition in distributing the airflow from the courtyard, although the better temperature is acquired due to the placement of vegetation and water body (see Figure 5 - temperature and velocity magnitude). The cool pocket is created near the building envelope, under the vegetation, and water body.

Envelope Scenario 2 provides a better opportunity for a cool pocket to be created. The area with a cool temperature near the building is larger and distributed more even compared than Envelope Scenario 1. The air flows better from the courtyard to the back part of the building (left side). This is significantly created by the subtraction of the middle part of the building which allows the air to flow and create more cool pockets (see Figure 6, near ground observation). Better distribution of cool pocket compared than heat pocket and also continuous air flow is created. In this case, the possible humidity that is created by the water vapour from the water body or vegetation can be reduced. The desired thermal condition is better achieved through this scenario.

\section{ENVELOPE SCENARIO 2}
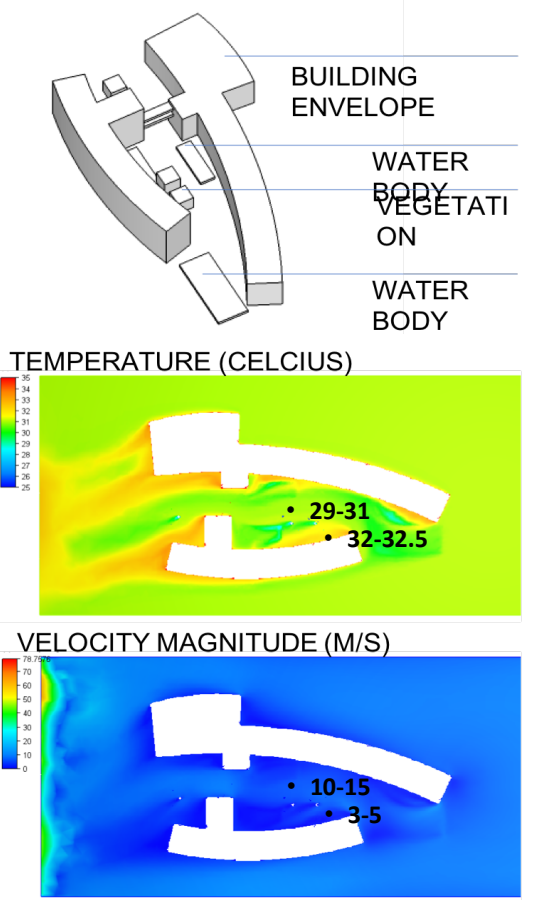

Fig. 6. Scenario 2 simulation based on second building envelope

Through two scenarios, learned that it is essential to create a passage for air to flow uninterruptedly. This effect is strengthened when the configuration is developed into the third scenario. Since the openings area for the wind to pass through the courtyard space is bigger - not only from right to left side but also both up or down side, the wind flow is now significantly improving the thermal condition of the courtyard space. Besides, the configuration of the water bodies and vegetation also contributes to this improvement, indicating a change which turns the building's courtyard space condition from heat island into a cool pocket (see Figure 7).

Architecture plays important part in this scenario by creating porous building geometry. Seen in the ground level observation, the courtyard is provided with more evenly distributed cooler temperature and air flow. Although the heat still happens nearby the building envelope on a higher level, higher air velocity occurs. This opens a possibility of further investigation in designing a cool pocket on building façade and as well the integration of vegetation and water body. 

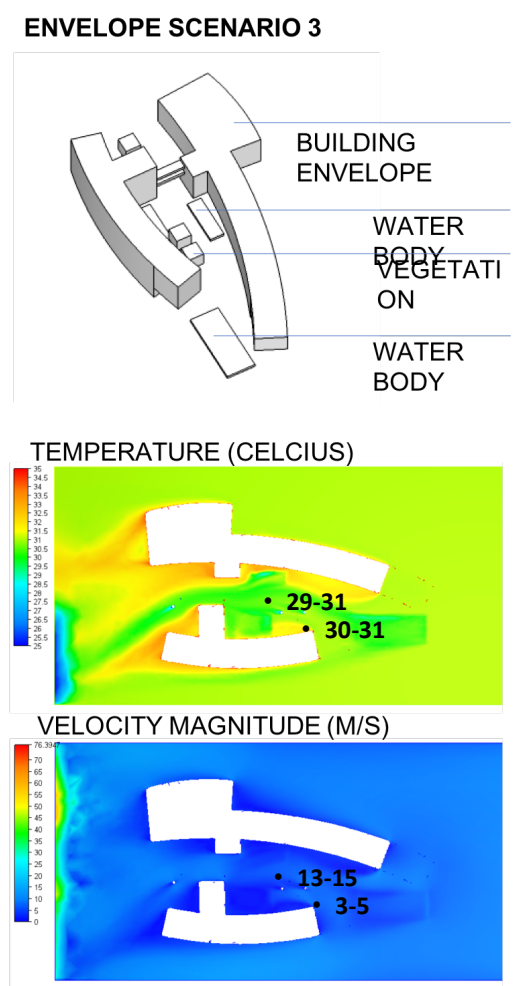

Fig. 7. The third scenario simulation that indicates the most preferable cool pocket creation

\section{Concluding Remarks}

Scenario-based simulation is an approach to designing cool pocket based on evidence. The scenarios are built on the ecological understanding that recognizes the various climatic attributes within a context and their complex interaction with the developed physical arrangements, particularly building envelope, vegetation and body water. In this case, CFD software acts as the tool to visualise the process. In this study, CFD Simulation is proven to be significant in order to show the interaction of elements, especially climatic attributes in the complex ecological system.

Through different scenarios, it is shown that the temperature and air flow act differently in response towards the arrangements of building envelope, vegetation, and body water. The scenarios are developed to achieve the desired thermal condition within the context. This implies a dynamic interaction between them can be created. In this case, architecture plays a vital role in creating cool pockets through mediation and manipulation, against the heat island phenomenon, which in this study turns out to be the cool pocket itself.

As a learning process, the scenario-based simulation shows the importance of ecological understanding as part of architectural education and design. It is also a critical phase in developing design strategies. The flexibilities in developing scenarios are provided and this can be applied as well as a learning module in the field of design and research.

\section{References}

1. Q. Chen, J.EnBuild 36 (2004)

2. M. Addington, The Unbounded Boundary in Thermodynamic Interactions, p. 82, NY: Actar Publisher (2017)

3. D.P. Saginatari, P. Atmodiwirjo, SHSConf 41, 06002 (2018)

4. Andrew C. Chui, et.al., J.Uclim 24 (2018)

5. Levermore, G., et al., J.Uclim 24 (2018)

6. R.C.Estoque, Y. Murayama, S.W.Myint, J.Scitotenv 577 (2016)

7. S. Murakami, J.Fluid Dynamics Research 38 (2006)

8. J.S. Sadar, Interiority 1 (2018)

9. J. Allegrini, J. Carmeliet, J.Uclim 21 (2017)

10. J. Deng, J., N. Wing, N. H., X. Zheng, J.Proeng 169 (2016)

11. D. Song, S. Han. J.Proeng 169 (2016).

12. H. Jin, T. Shao, T., R. Zhang, J.Egypro 134 (2017)

13. W.E. El-Bardisy, M. Fahmy, G.F. El-Gohary, J.Sbspro 216 (2016).

14. FICCI, Leapfrogging to Education 4.0 : Student at the Core (2017)

15. S. Kaijima, R. Bouffanais, K. Willcox, CAADRIA 18th (2013)

16. P.A. Mirzaei, F. Haghighat, J.Buildenv 45 (2010)

17. Y. Toparlara, B. Blockena, B. Maiheub, G.J.F. van Heijstd, J. Erser 80 (2017)

18. B. Stone Jr, M.O. Rodgers, Michael, JAP 67, 2, (2001) 